\title{
Une dynastie de parfumeurs du roi : les Gallois/ Huet et la fabrique des apparences de la cour à la ville, $1686-1789$
}

A dynasty of king's perfumers: the Gallois/Huet and the factory appearances, from the court to the city, 1686-1789

\section{Catherine Lanoë}

\section{OpenEdition} Journals

Édition électronique

URL : http://journals.openedition.org/artefact/6022

DOI : $10.4000 /$ artefact.6022

ISSN : 2606-9245

Éditeur :

Association Artefact. Techniques histoire et sciences humaines, Presses universitaires du Midi

\section{Édition imprimée}

Date de publication : 15 juillet 2020

Pagination : 317-348

ISBN : 978-2-8107-0691-4

ISSN : 2273-0753

\section{Référence électronique}

Catherine Lanoë, « Une dynastie de parfumeurs du roi : les Gallois/Huet et la fabrique des apparences de la cour à la ville, 1686-1789 », Artefact [En ligne], 12 | 2020, mis en ligne le 21 décembre 2020, consulté le 23 décembre 2020. URL : http://journals.openedition.org/artefact/6022 ; DOI : https:// doi.org/10.4000/artefact.6022

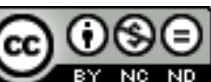

Artefact, Techniques, histoire et sciences humaines est mise à disposition selon les termes de la Licence Creative Commons Attribution - Pas d'Utilisation Commerciale - Pas de Modification 4.0 International 


\section{Une dynastie de parfumeurs du roi : les Gallois/Huet et la fabrique des apparences de la cour à la ville, 1686-1789}

\section{Catherine Lanoë}

\section{Résumé}

Patiente et méthodique, la constitution d'un corpus d'archives manuscrites, issues du fonds du Minutier central des Archives nationales, avait déjà permis de le mettre au jour : établie rue de l'Arbre-Sec à « l'Orangerie royale », la dynastie de parfumeurs Gallois/Huet a assuré pendant plus d'un siècle l'approvisionnement du roi de France et de la cour en marchandises de parfumerie et de cosmétique. En s'appuyant sur un ensemble documentaire plus vaste encore, composé d'inventaires après décès, de contrats de mariage, de vente et de bail, articulant histoire sociale, histoire du corps et histoire des savoirs, cet article entend reconstituer la destinée exceptionnelle de cette entreprise artisanale parisienne d'Ancien Régime et dessiner le profil de l'artisan parfumeur commensal. Pour autant, la représentativité de ce modèle et la singularité de cette histoire sont aussi interrogées ici puisqu'elles peuvent être inscrites, d'une part, dans le panorama plus vaste du métier de gantier-parfumeur aux xvII ${ }^{e}$ et $x V I I I^{e}$ siècles et, d'autre part, dans celui des évolutions de la fabrique des apparences de la cour à la ville, depuis le règne de Louis XIV jusqu'à la Révolution.

\section{Mots-clés}

Huet, « Orangerie royale », parfumeurs, cour, gants, fleur d'oranger

99 Catherine Lanoë, « Une dynastie de parfumeurs du roi : les Gallois/Huet et la fabrique des apparences de la cour à la ville, 1686-1789 », Artefact, 12, 2020, p. 317-348. 


\section{$A$ dynasty of king's perfumers: the Gallois/Huet and the factory appearances, from the court to the city, 1686-1789}

\section{Abstract}

Patient and methodical, the constitution of a corpus of handwritten archives, from the collection of the Minutier central of the National Archives, had already made it possible to bring it to light: established in rue de l'Arbre-Sec at the "Royal Orangery", the dynasty of Gallois/Huet perfumers ensured for more than a century the supply of the king of France and the court with perfume and cosmetic goods. Drawing on an even larger documentary collection, comprising inventories after death, marriage contracts, sale and lease, articulating social history, history of the body and history of knowledge, this article intends to reconstruct the exceptional destiny of this Parisian artisanal company of the Old regime and draw the profile of the artisanal perfumer commensal. However, the representativeness of this model and the singularity of this history are also questioned here since they can be inscribed, on the one hand, in the wider panorama of the profession of glove-maker and perfumer in the seventeenth and eighteenth centuries and, on the other hand, in that of the developments in the appearance of the court in the city, from the reign of Louis XIV until the Revolution.

\section{Keywords}

Huet, « Orangerie royale », perfumers, court, gloves, orange blossom 


\section{À Sophie Lefay}

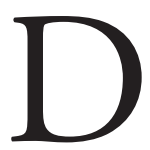

epuis les travaux fondateurs de Daniel Roche et de Philippe Perrot, les notions de « culture des apparences » et de « travail de l'apparence » se sont installées dans le paysage historiographique français ${ }^{1}$. De multiples études, dans lesquelles les historiens et les conservateurs des musées ont parfois ouvré de concert, ont ainsi été réalisées sur les différents objets qui permettent cette fabrique du corps socialisé et politisé, de sorte qu'ils échappent totalement désormais aux registres de la " frivolité » et de la "futilité » dans lesquels l'histoire de la culture matérielle les avait parfois inscrits ${ }^{2}$. Aux côtés des dispositifs vestimentaires qui figurent en bonne place parmi ces travaux et qui ont donné lieu à plusieurs ouvrages de synthèse, différentes parures du corps ont aussi été appréhendées, parmi lesquelles figurent les coiffures et les perruques, les produits cosmétiques et ceux de parfumerie ${ }^{3}$. Dans le cadre des cours de l'Europe moderne, et surtout de celle de Louis XIV, ces différents artefacts ont parfois été embrassés ensemble, car ils relèvent de " techniques du corps" qui forment système et traduisent la production de normes du paraitre adaptées à la mise en œuvre d'un contrôle politique et social, progressivement intériorisé par les élites ${ }^{4}$. Au cours du XvIII ${ }^{e}$ siècle, cependant, avec les inflexions incessantes de la mode et l'élargissement social du marché parisien du vêtement et de la beauté, ces constructions se libèrent partiellement des prescriptions, se font plurielles, donnant ainsi plus de place à l'expression de l'individualité des consommateurs et stimulant la créativité de tous les " artisans du corps $"^{5}$.

Ces derniers, pourtant, n’ont pas encore donné lieu à de nombreux travaux ${ }^{6}$. Surtout, leurs métiers et les savoir-faire qu'ils ont mis en œuvre n'ont été que trop rarement mis au jour par l'exploitation de sources qui permettent vraiment de les éclairer et de les comprendre, au profit de sources rattachées à l'histoire de la consommation, ou bien de sources savantes qui n'en donnent qu'une image partielle et partiale, souvent dévalorisante.

\footnotetext{
1. Roche, 1989 ; Perrot, 1984.

2. Braudel, 1979, p. 288 et 290.

3. McNeil, 2017 ; Bruna et Demey, 2018.

4. Mauss, 1936 ; Arrizoli et Gorguet, 2009 ; Paresys et Coquery, 2011 ; Da Vinha, Lanoë et Laurioux, 2011.

5. Cavallo, 2007.

6. Castres, 2016 (thèse à paraitre).
} 
Au contraire, comme l'ont montré quelques ouvrages récents, parfois éloignés de l'univers des apparences, les techniques artisanales, révélées dans de multiples archives - dossier de faillite, registres de compte, inventaires après décès... - se signalent par de fortes capacités d'adaptation et d'innovation, de véritables savoirs, en particulier dans le contexte du développement de la consommation au XVIII ${ }^{\mathrm{e}}$ siècle ${ }^{7}$.

Les pratiques des gantiers-parfumeurs en témoignent : l'exploitation d'un vaste corpus d'archives a donné la possibilité de reconstituer les gestes de leur métier dans la fabrication des différents cosmétiques en usage aux $\mathrm{XVII}^{\mathrm{e}}$ et $\mathrm{XVIII}^{\mathrm{e}}$ siècles ${ }^{8}$. À la faveur de ce travail, certains de ces artisans avaient été identifiés pour avoir en charge l'approvisionnement du roi et de la cour, mais le caractère général du propos n'avait pu leur accorder l'attention qu'ils méritaient'. Précisément, parmi tous les fournisseurs des souverains successifs, la récurrence d'un nom de famille, les Huet, et celui d'une boutique, "l'Orangerie royale ", s'était déjà avérée frappante, car l'enquête dans les archives du Minutier central des notaires parisiens avait permis de retrouver un inventaire du fonds de commerce de cette boutique à cinq dates différentes entre 1686 et $1789^{10}$. Fait très exceptionnel pour une entreprise artisanale de l'Ancien Régime, que traduit un corpus d'archives exceptionnel, la boutique a été tenue sans discontinuité par une véritable dynastie de gantiers-parfumeurs du roi entre la fin du XVII ${ }^{\mathrm{e}}$ siècle et la fin du XVIII ${ }^{\mathrm{e}}$ siècle.

Relevant en partie seulement de la prosopographie ou de la microstoria, puisque le destin de cette famille d'artisans peut être inscrit dans un panorama plus vaste susceptible d'interroger sa représentativité, l'article proposé ici repose donc sur l'analyse d'un ensemble de documents rassemblés

7. Hilaire-Pérez, 2013 ; Labreuche, 2011.

8. Lanoë, 2008. Sur les parfumeurs du roi aux XVII ${ }^{\mathrm{e}}$-XVIII ${ }^{\mathrm{e}}$ siècles, nous signalons la thèse en cours d'Alice Camus sous la direction de Lucien Bély (Paris-Sorbonne).

9. Lanoë, 2008, p. 258-262.

10. Lanoë, 2008, p. 62. Il s'agit 1) de l'inventaire qui est dressé lors d'une vente réalisée par Michel Gallois à son neveu François Huet le 12 juillet 1686 (AN, MC XCVII/72) ; 2) de celui qui est dressé au moment de l'inventaire après décès de ce dernier le 23 septembre 1711 (AN, MC XLIX/456); 3) d'un état du fonds de boutique établi le 29 décembre 1732 lorsque Geneviève de Peyras, épouse de François Huet, délaisse ce même fonds à son fils François Huet (AN, MC XLIX/547) ; 4) de l'inventaire dressé au moment de la mort de ce dernier le 26 novembre 1754 (AN, MC XC/382); 5) de celui qui est établi le 28 septembre 1789 lorsque meurt Marie-Bénédicte Poton, épouse de Claude-François Prévost, veuve en premières noces de François Huet (AN, MC XLIII/556). 
et partiellement traités autrefois, que les besoins de l'enquête ont enrichi de nouvelles archives, pour la plupart issues du fonds du Minutier central, contrats de bail et contrats de mariage en particulier. Histoire sociale, histoire du corps et histoire des savoirs se conjuguent ici, dans la mesure où cette entreprise artisanale constitue un observatoire de choix pour saisir le caractère spécifique des sensibilités et des constructions de l'apparence dans les milieux privilégiés de la cour entre le XvII ${ }^{\mathrm{e}}$ et le XVIII ${ }^{\mathrm{e}}$ siècle, auxquelles la dynastie des Gallois/Huet s'est efforcée de répondre en articulant techniques traditionnelles et innovations.

Après avoir reconstitué l'histoire de la boutique et celle de l'entreprise ellemême, en montrant les inflexions que subit le modèle de l'artisan commensal au contact d'un marché qui s'étend au-delà de la cour et s'avère toujours plus concurrentiel, un second temps sera consacré à l'étude des objets de parure et des formes que revêtent la culture du corps et la fabrique des apparences des privilégiés de la cour à la ville, tandis que le dernier temps de la réflexion, inscrit à l'échelle d'un article, la poudre pour les cheveux, permettra de mesurer l'évolution de la consommation d'un produit de beauté et de mettre au jour les savoirs du métier.

\section{L'Orangerie royale, une entreprise artisanale familiale florissante et pérenne}

En 1692, Le Livre commode contenant les adresses de la ville de Paris... recense la boutique de l'Orangerie royale parmi celles auprès desquelles le consommateur peut se procurer "en détail de bonne eau de fleurs d'oranges ", mais la boutique existe en réalité depuis 1686 au moins ${ }^{11}$. Sans nul doute, son appellation est liée à l'engouement dont ce parfum est alors l'objet à la cour de Versailles et, peut-être, un hommage particulier à la seconde orangerie dont se dote le château à partir de $1683^{12}$.

11. Blégny, 1692, p. 98.

12. Perez, 2013 ; Da Vinha et Masson, 2015, p. 525. 


\section{Une boutique et son histoire}

Comme tous les notaires le précisent, l'Orangerie royale est située rue de l'Arbre-Sec, paroisse Saint-Germain-l'Auxerrois, " presque vis-à-vis la rue Bailleul ». Une enquête menée dans les archives et sur le terrain permet d'affirmer qu'elle se trouvait à l'emplacement du numéro 50 actuel (Fig. LI, cahier couleur) $)^{13}$.

La boutique fait alors partie d'un corps de logis de devant, située à gauche d'une porte cochère qui permet d'accéder à une cour et à un second corps de logis à l'arrière de la parcelle. Jusqu'en 1731, l'ensemble appartient aux héritiers du notaire Jean Chapellain III, date à laquelle ceux-ci se décident à vendre, après l'avoir fait visiter et estimer deux fois par la Chambre des bâtiments. Jugée " très caduque et à la veille d'être rebâtie " de l'avis du second expert, ce qu'elle ne sera jamais apparemment au cours du siècle, la maison n'en est pas moins estimée 40000 livres en $1728^{14}$. Trois ans plus tard, avant même d'être mise aux enchères devant les Criées du Châtelet, elle trouve un acquéreur qui en propose 45000 livres, réglées dès la signature du contrat de vente le 9 août 1731 en louis d'or et d'argent ${ }^{15}$. L'acheteur, en effet, n'est autre que Jean Quentin, baron de Champlost, « écuyer et

13. Les informations des actes notariés croisées à celles que fournit l'estimation de la maison en 1731 (AN, Z1J/623) ont été confrontées à une enquête de terrain. De multiples indices concordent. Les " quatre travées de profondeur " du corps de logis de devant sont encore bien visibles au plafond de la boutique actuelle, tandis que sa longueur (53,5 pieds) et sa largeur (19,5 pieds) mesurées en 1731 correspondent à celles qui peuvent être mesurées aujourd'hui, soit environ 18 mètres de long sur 6,30 de large. Surtout, l'estimation de 1731 signale la présence d' ' un étage de caves sous et en toute l'étendue du corps de logis sur rue, édifices; en aile à gauche de la cour sous parties d'icelles » qui ont pu être identifiées sur place. Les caves situées sous la boutique étaient et sont encore accessibles, d'une part par une " potoyère ", c'est-à-dire une descente en marches de pierre (Carbonnier, 2006, p. 378), localisée dans la cour sous un escalier, et d'autre part par une trappe aménagée dans la boutique, dont un employé actuel nous a dit avoir connaissance.

14. Cette estimation est un peu inférieure au denier 20 qui constitue le coefficient par lequel sont multipliés les loyers pour obtenir le prix de vente des maisons, puisqu'en effet à cette date le loyer du corps de logis de devant est de 1200 livres par an, tandis que celui du corps de logis de derrière est de 1000 livres (en 1731), ce qui donnerait 44000 livres (Voir Lyon-Caen, 2015, p. 63). Sans doute, l'état de vétusté constaté de la maison l'explique.

15. ET. LXIV/301. Le déroulement de la procédure de mise aux enchères de la maison est conforme à ce qui a été identifié par ailleurs (affiches, publications concernant la vente) et l'absence d'enchère confirme la faiblesse du nombre d'acheteurs potentiels (Béguin, Lyon-Caen, 2018). L'offre de 45000 livres du baron de Champlost rejoint sensiblement la règle du denier 20 qui structure le marché immobilier parisien. 
premier valet de garde-robe du roi demeurant à Paris au Louvre ", fils de Jean Quentin et neveu de François Quentin, dit La Vienne - qui tous deux avaient exercé la fonction de barbier de Louis XIV entre 1669 et 1690 et pour Jean celle de perruquier également -, avant d'accéder aux prestigieuses charges de premier valet de chambre du roi pour François et de premier valet de garde-robe pour Jean ${ }^{16}$. Même si la volonté d'investir dans ce quartier parisien, déjà connu pour ses boutiques de luxe, peut expliquer l'offre du baron, jugée " très avantageuse " selon l'expert de 1731, on ne peut douter que l'intérêt de cette acquisition tient aussi à la présence de la très réputée boutique de l'Orangerie royale ${ }^{17} \ldots$

Pendant plus d'un siècle, la dynastie s'acquitte de son loyer à ces propriétaires successifs, puis à Marie-Louis Quentin de Champlost, fils de Jean, qui hérite de la maison lors de son mariage en 1740, tous intimement liés au milieu de la cour et au service du roi. Quoique le loyer annuel augmente de 1100 livres en 1686 à 1300 livres en 1776, les locaux de l'entreprise restent les mêmes jusqu'en $1779^{18}$. Les descriptions notariées croisées à l'analyse de l'estimation de 1731 permettent d'identifier dans ce corps de logis de devant une boutique ouverte sur la rue, fermée par un châssis vitré, prolongée par une salle/arrière-boutique, l'une et l'autre planchéiées ${ }^{19}$. Elles sont séparées par une cloison partiellement vitrée, tandis que l'arrière-boutique est fermée à l'arrière par une croisée qui éclaire la pièce par la cour. Cette quête de lumière, conjuguée à la présence d'une cheminée, révèle que l'arrière-boutique sert d'atelier au parfumeur. Quant à la boutique, de forme rectangulaire, sa taille est difficile à évaluer. S’il est possible d'estimer sa profondeur à huit mètres, peut-être douze, sa largeur en revanche ne peut en excéder quatre, compte tenu des deux mètres que représentent la porte cochère et le passage qui lui fait suite vers la cour ${ }^{20}$. Un escalier permet de se rendre aux étages, composés de chambres et/ou de magasins, affectés et désignés différemment selon les dates, qui permettent de loger la famille de l'artisan, le personnel de la boutique ou des sous-locataires, ou d'entreposer la marchandise. L'artisan dispose aussi de l'accès à la cour,

16. Da Vinha, 2004.

17. Coquery, 2011, p. 142-144.

18. Sur l'augmentation des loyers, voir Lyon-Caen, 2015, p. 56.

19. Sur la variété des boutiques parisiennes, voir Carbonnier, 2006, p. 353-359.

20. AN, Z1J/623. Nous remercions Robert Carvais (Centre de théorie et analyse du droitUMR 7074-Université Paris Nanterre) pour son aide dans la transcription et la compréhension de ce document. 
partiellement envahie par " plusieurs petits édifices », et « la commodité du puits ", bien utiles pour ses activités professionnelles. Enfin, les notaires et le greffier de la Chambre des bâtiments soulignent l'existence de plusieurs caves qui permettent d'entreposer du bois et du charbon, nécessaires à la confection des feux destinés aux opérations du métier (voir note 13). En 1779, dans le cadre d'une restructuration de l'entreprise, les locaux s'agrandissent considérablement : par transport de bail, Marie-Bénédicte Poton, veuve de François Huet fils et épouse de Claude-François Prévost, devient également locataire du corps de logis de derrière, le tout pour un loyer de 2700 livres par an (Fig. 1).

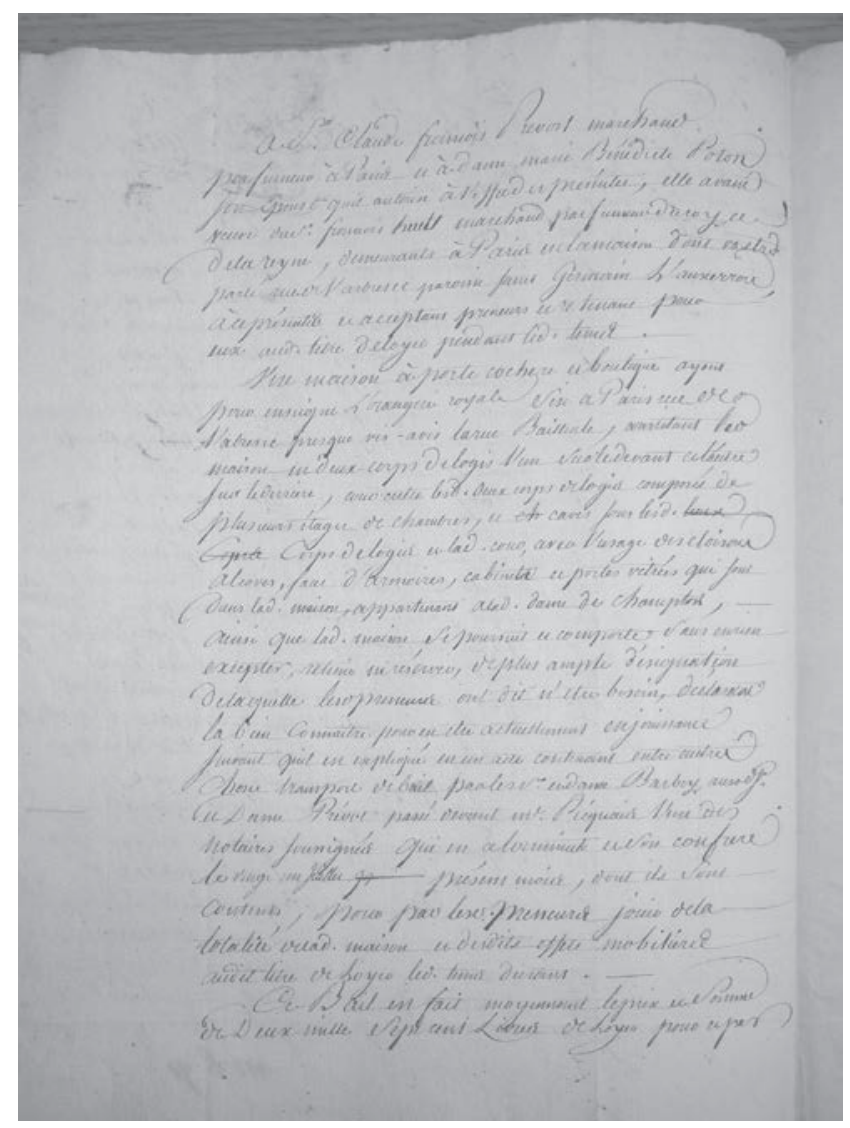

Fig. 1. - Extrait du contrat de bail du 29 juillet 1779

AN, MC LVI/241-242, fol. 2.

Archives nationales, photographie Catherine Lanoë 
Manifestement, la pérennité exceptionnelle de la boutique s'est accompagnée d'une indiscutable prospérité que reflète déjà le montant de cette charge locative. Surtout, elle atteste de la part de tous les acteurs de l'entreprise, et en particulier des deux femmes qui en ont eu la gestion à la mort de leur époux, Geneviève de Peyras entre 1711 et 1732, puis MarieBénédicte Poton entre 1754 et 1767, la compréhension avisée des lois d'un marché de cour spécifique et changeant et la mise en œuvre de savoirs sociaux adaptés.

\section{Fournir la cour ou le savant calcul des avantages}

Tout au long du siècle, la dynastie tient en partie le marché de la cour. Dès 1686 en effet, François Huet père peut se réclamer du double statut de «marchand parfumeur... privilégié ordinaire du roi » et de «marchand parfumeur privilégié suivant la cour », qui fait de lui un artisan participant à la commensalité et au service du roi, mais aussi à l'approvisionnement de grandes maisons aristocratiques ${ }^{21}$.

Effectivement, comme le révèlent par exemple six mémoires de marchandises établis entre 1708 et 1711 - sommés ensemble à 7189 livres, auxquels s'ajoutent 1149 livres pour marchandises fournies " à sa majesté " et "pour le Dauphin » en 1711 -, Louis XIV, approvisionné par le duc de La Rochefoucauld, grand maître de la garde-robe et confident du roi, consomme certains articles de l'Orangerie royale ${ }^{22}$. Pour toutes ces fournitures, la veuve déclare avoir " reçu acompte de 2189 livres, 18 sols », en différents paiements échelonnés depuis 1709, jusqu'à l'obtention d'un billet de 500 livres après le décès du parfumeur. Quelques décennies plus tard, un autre couple royal fait partie des clients que Claude-François Prévost recense dans son état des dettes actives, en particulier pour des achats de lavande (Fig. 2) ${ }^{23}$.

21. Laverny, 2000 ; Da Vinha et Masson, 2015, p. 458-459.

22. Da Vinha, 2004, p. 640.

23. AN, MC XLIII/556. 


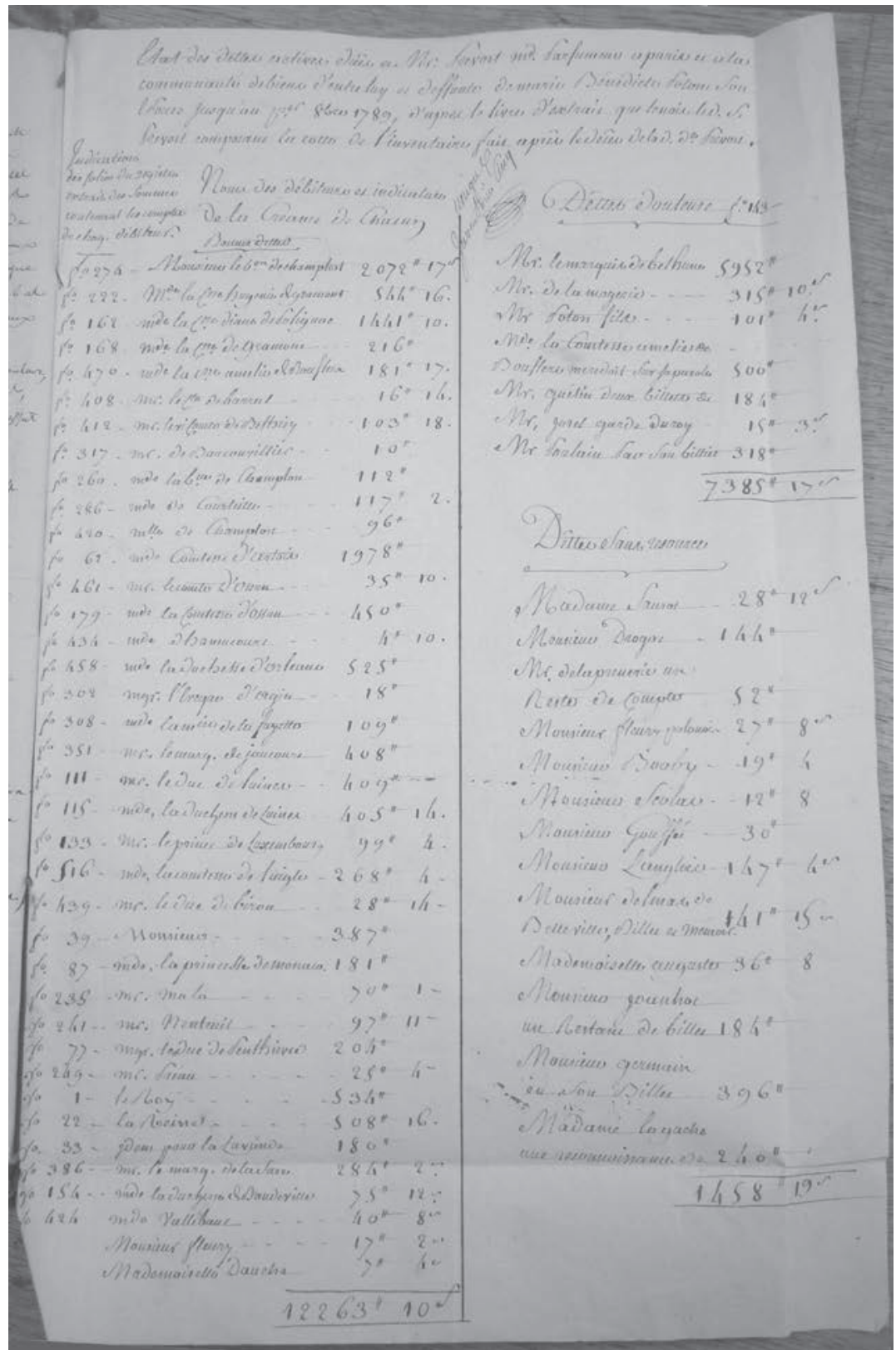

Fig. 2. - État des dettes actives dues à M. Prévost..., 28 septembre 1789

AN, MC XLIII/556, fol. 40.

Archives nationales, photographie Catherine Lanoë 
À tout le moins, le couple royal est mentionné dans la catégorie des "bonnes dettes ", ce qui est loin d'être le cas de nombreux clients de la boutique au cours du siècle. La chose est connue en effet : la plus haute noblesse s'acquitte souvent à retardement de ses dépenses somptuaires, quand elle s'en acquitte d'ailleurs ${ }^{24}$. En l'occurrence, en 1711, Geneviève de Peyras, qui connaît parfaitement sa clientèle, renonce d'elle-même à entreprendre quelque démarche que ce soit contre les débiteurs "qu'elle croira et apprendra être insolvables ».

Pour autant, François Huet et son épouse ne sont pas sans ressource face à ces impayés. Comme marchand privilégié, le parfumeur est enregistré auprès de la Prévôté de l'Hôtel. Le dépôt de plaintes devant cette juridiction, ou dans une moindre mesure devant celle du Châtelet de Paris, lui permet d'obtenir en sa faveur de multiples sentences condamnant au paiement ses clients indélicats, qui s'acquittent alors de leurs dettes, bien souvent sous la forme de billets ou de contrats de constitution de rentes viagères ${ }^{25}$. Ainsi, quoique l'argent ne rentre pas toujours sous la forme d'espèces sonnantes et trébuchantes, la Prévôté de l'Hôtel assure à l'entreprise une protection essentielle à sa survie. Elle construit dans l'espace curial un équilibre qui garantit la pérennité du marché, puisque les condamnés, débarrassés de leurs dettes, peuvent alors reprendre leurs achats auprès des mêmes fournisseurs. Geneviève de Peyras en est parfaitement consciente, puisque dans les tout premiers jours qui suivent la mort de son mari, elle s'acquitte des 420 livres qui lui permettent de racheter le titre pour devenir à son tour "marchande gantière-parfumeuse privilégiée suivant la cour ".

Autorisée par le statut et la production de François Huet, l'inscription dans le milieu curial se conjugue de manière heureuse avec l'appartenance de son épouse à l'univers des officiers de finance anoblis et enrichis. Dotée de 6000 livres - qui permettent le rachat du fonds de boutique de Michel Gallois - celle-ci est fille d'un ancien "Conseiller du roi payeur des gages de Messieurs les officiers de l'élection de Paris ", et sœur d'un " contrôleur général des rentes sur les aides et gabelles ». Cette conjonction offre au couple des perspectives d'enrichissement, via des investissements ou des placements qui s'articulent aux rentrées d'argent, à son manque ponctuel et aux logiques de thésaurisation. Ainsi, peu avant son décès, le parfumeur

24. Coquery, 1998, p. 163-178.

25. Da Vinha et Masson, 2015, p. 585-586. 
a fait l'acquisition de différents terrains parisiens, l'un de 816 toises carrées au lieu de la Grange-Batelière, l'autre de 700 toises rue de Richelieu, sur lesquels il fait construire des maisons, louées ensuite à des artisans, tandis que son épouse souscrit sur la tête de ses enfants plusieurs contrats de constitution de rentes sur les aides et gabelles durant les années 1720 et investit dans des actions de la compagnie des Indes en 1737. Entre 1732 et son décès en 1738 , elle est ainsi en mesure de faire bénéficier chacun de ses cinq enfants vivants - sur les onze qu'elle a eus - de 12000 livres, dont 8000 versées en espèces pour la dot de chacune de ses trois filles, quand François II, l'aîné de ses fils, se voit céder le fonds de boutique et les dettes actives de l'entreprise pour cette même somme.

\section{Le règne de la concurrence et la restructuration de l'entreprise}

En 1732, François II reprend l'entreprise à son compte. Comme l'attestent plusieurs documents, il n'est alors plus revêtu du titre de « marchand privilégié suivant la cour ", conservant en revanche celui de " marchand parfumeur ordinaire du roi et de la reine ", un statut honorifique qui se donne à voir à l'étalage de la boutique avec les «trois tapis » qui portent des « fleurs de lys aux armes du roi et de la reine ». Il n'est cependant pas le seul à pouvoir le faire, car dès le milieu du siècle, à Versailles ou à Paris dans ce même quartier, la concurrence entre les gantiers-parfumeurs fournisseurs du roi et de la cour est grandissante. Ainsi, François Huet puis sa veuve doivent-ils faire face, par exemple, à celle de Joannis, "privilégié du roi " demeurant rue de l'Arbre-Sec, à celle de Michel Marganne, "parfumeur du roi " rue Saint-Honoré, puis à celle de Jean-Daniel Vigier " marchand mercier et parfumeur ordinaire du Roi ", installé au 11 de la rue du Roule, où lui succède en 1774 Jean-Louis Fargeon, parfumeur de la cour et de Marie-Antoinette. De surcroît, des fabricants toujours plus nombreux, corporés ou non, portés par la réputation et la qualité de leur production, tout autant voire davantage que par un titre prestigieux, parviennent à s'immiscer dans ce marché. La consommation parisienne des biens de l'apparence, enfin, partagée désormais entre les privilégiés et des catégories plus modestes, est aussi transformée par les incessants renouvellements de la mode, ce qui constitue un double appel d'air pour tous les « artisans du corps ", pour peu qu'ils adaptent leurs stratégies à ces nouvelles logiques. 
Mariée à François Huet en 1735, Marie-Bénédicte Poton fait sans doute l'expérience de ces changements et, peut-être, celle d'une prospérité moins éclatante, quoique le couple ait été en mesure d'investir 3600 livres dans une maison de campagne à Nanterre en 1754, de la meubler et d'y faire entreprendre diverses réparations. Cependant, même si la reine s'avère être une cliente fidèle - comme en témoignent quatre mémoires arrêtés par la duchesse de Villars pour l'année 1754 montant à 961 livres - et que la veuve parvient à se faire payer de 2000 livres dans les trois semaines qui suivent le décès de son mari, le rapport entre la valeur du fonds de boutique (4 704 livres), les dettes actives (5 592), les dettes passives (4 486) et les dettes actives douteuses (11 185 livres, sur lesquelles la veuve déclare pouvoir récupérer la moitié) laisse entrevoir une situation financière moins favorable que par le passé, des difficultés croissantes pour se faire payer par des aristocrates toujours plus dispendieux, que la veuve sait pourtant parfois insolvables, voire en faillite ${ }^{26}$.

L'équilibre de la première moitié du siècle dont avaient profité les premiers patrons de l'Orangerie royale n'est plus. Marie-Bénédicte Poton entreprend alors de restructurer l'entreprise pour l'adapter aux nouvelles logiques du marché. Cette restructuration se marque tout d'abord par son remariage en 1767 avec Claude-François Prévost, " marchand parfumeur demeurant rue de l'Arbre-Sec » - travaillant peut-être déjà à son service - alors âgé de 26 ans, quand elle doit en avoir près du double. Les termes du contrat de mariage révèlent une véritable association qui doit profiter à chacun, permettre de relancer l'affaire, sans préjudicier aux cinq enfants que la veuve a eus de François Huet. En effet, les biens de chacun des époux - 3000 livres de gains et épargne du côté de Claude-François et 24000 livres en marchandises, meubles, hardes du côté de Marie-Bénédicte - n'entrent pas dans la communauté, qui ne sera constituée que de ce qu'ils acquerront ensemble, tandis que les deux époux ne peuvent se faire de donation au dernier vivant qu'à la condition qu'aucun enfant ne naisse du mariage. Claude-François Prévost, fils de laboureur de Franche-Comté, parfumeur sans fonds ni boutique, est désormais revêtu du titre « de marchand parfumeur du roi et de la reine » et se retrouve à la tête d'une entreprise installée et réputée, qu'il contribue à relancer.

26. Coquery, 1998, p. 119-126. 
De fait, en 1779, par le transport de bail évoqué plus haut, les locaux de l'entreprise sont agrandis au second corps de logis. Il s'agit en effet d'abriter un stock bien différent des précédents, dont la valeur atteint 4360 livres seulement en 1789, soit trois fois moins que celui que rachète François Huet à son oncle en 1686, sans même tenir compte de l'inflation. L'immobilisation du capital est ainsi limitée et cette stratégie s'avère adaptée puisque l'artisan supporte des impayés toujours plus nombreux : sur les 21106 livres de dettes actives, près de 9000 sont estimées douteuses, voire sans ressources (Fig. 2). Ce stock, en revanche, est une illustration de l'économie de la diversité qui s'est alors imposée. Il est constitué d'un nombre beaucoup plus grand d'articles que par le passé, estimés à des prix variables dont certains modiques peuvent convenir à une clientèle modeste, et qui se répartissent de manière rationnelle dans les différents magasins et la boutique. Cette dernière justement, quoique l'enseigne de l'Orangerie royale ne semble pas y pendre à la date du 28 septembre 1789 afin d'éviter, peut-être, une publicité qui n'était déjà plus dans l'air du temps, a aussi donné lieu à des aménagements récents, destinés à séduire et attirer la clientèle, à l'accueillir comme dans un salon ${ }^{27}$. Ainsi, les deux comptoirs de la boutique sont de " menuiserie de chêne neuve ", précise par deux fois le notaire, tandis que l'exiguïté et la pénombre relative de la boutique sont compensées par de nombreuses menuiseries peintes en blanc et un grand panneau de glaces. L'empire de la mode et du commerce, la place essentielle qu'y tiennent les femmes désormais sont aussi sensibles dans la répartition sexuée des postes de travail. Claude-François Prévost emploie deux garçons, les frères Féry, logés au dernier étage et payés 100 livres pour six mois, mais l'entreprise dispose aussi d'une fille de boutique, JeanneBaptiste Maignier, qui occupe une chambre au premier étage et dont le salaire est le même que celui des employés fabricants.

\section{Cultures du corps et fabrique des apparences}

Courant sur plus d'un siècle, l'histoire de l'Orangerie royale et de ses dirigeants successifs, croisée à l'analyse des cinq états du stock permettent d'observer avec précision les sensibilités et les normes sociales qui président

27. Walsh, 2000; Velut, 2000. 
à la fabrique des apparences, essentiellement dans les milieux privilégiés, de la cour à la ville. De véritables inflexions se dessinent entre 1686 et 1789 , car si la valeur estimée du fonds de boutique diminue au moins de moitié entre ces deux dates, la nature même des marchandises n'en est pas moins profondément transformée.

\section{Un univers domestique parfumé}

De manière tout à fait évidente, la constitution des trois plus anciens inventaires du fonds de la boutique $(1686,1711,1732)$ révèle le poids essentiel des marchandises de parfumerie. Aux côtés des essences et des eaux de senteur conservées en diverses sortes de bouteilles ou, en particulier lorsqu'il s'agit de la fleur d'oranger, dans des estagnons - c'est-à-dire des récipients de métal étamé contenant plusieurs dizaines de pintes les gants et les peaux, les éventails, les objets parfumés constituent l'essentiel du stock, avec les poudres pour les cheveux. Tous ces articles ont pour dénominateur commun d'avoir été l'objet de l'imprégnation par un parfum, puisque depuis 1614 les gantiers ont obtenu à l'exclusion de tous les autres artisans tenant boutique, tels que les merciers en particulier, le titre de parfumeurs ${ }^{28}$. Comme Stanis Pérez l'a bien montré, si l'eau de fleur d'oranger a les faveurs de la cour à l'époque de Louis XIV, les parfums n'entrent pas alors en contact direct avec le corps. Il revient alors à différents objets et aménagements de dissiper la précieuse fragrance dans l'espace domestique : aspersion à la seringue dans les appartements du roi, installation d'orangers dans les chambres, dissémination de leurs fleurs autour des lits, dispersion de sachets parfumés et autres articles que l'on dit parfois " piqués d'odeur " ${ }^{29}$.

Effectivement, le stock des trois premiers représentants de la dynastie est composé de nombreuses marchandises de cette nature, parmi lesquels se comptent bourses à jouer, sachets, boîtes à perruques, corbeilles (destinées à recevoir habits, gants et garnitures diverses), auxquels s'ajoutent, parfois assortis entre eux, les toilettes - c'est-à-dire les pièces de toile qui couvrent les tables surmontées du miroir devant lesquelles se déroulent les différents apprêts du visage - et les déshabillés qui, de l'avis expert de Simon Barbe, sont "des portefeuilles de carton ", parfois de véritables coffres, doublés

28. Lanoë, 2008, p. 63.

29. Pérez, 2013, p. 108-110. 
Catherine Lanoë

de différentes étoffes parfumées et qui accueillent pour la nuit les « coiffes, rubans et menu linge des dames, lorsqu’on les déshabille» (Fig. 3) ${ }^{30}$.

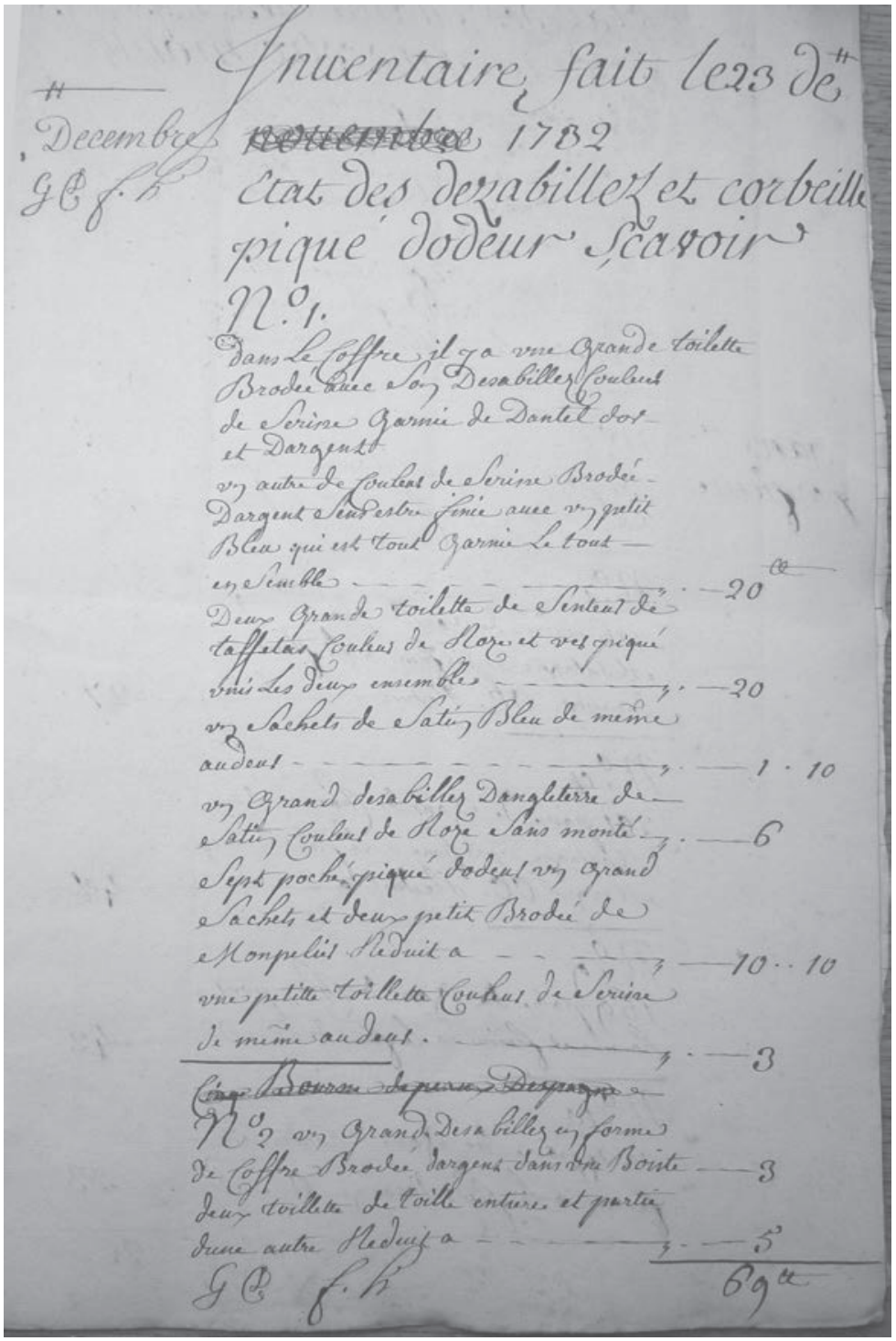

Fig. 3. - Extrait de l'inventaire dressé par Geneviève de Peyras, 23 décembre 1732

AN, MC XLIX/547, fol. 6.

Archives nationales, photographie Catherine Lanoë

30. Barbe, 1992 [1699], p. 110-111. 
Ces différents objets permettent à ceux qui les acquièrent de composer dans les grandes maisons princières et jusque dans la chambre du roi, par l'intermédiaire du grand maître de la garde-robe, un univers domestique parfumé. Celui-ci, dans le cadre des conceptions néohippocratiques en vigueur, atteste la volonté prophylactique d'éloigner les mauvaises odeurs, de construire une atmosphère olfactive que l'on imagine salubre et favorable à la santé du prince ${ }^{31}$. Sur le plan social, cette conception et ces ajustements renvoient largement à l'héritage des pratiques des parfumeurs-valets de chambre, des commensaux traditionnels dans les maisons princières dont les attributions sont de plus en plus bousculées par la professionnalisation du métier, comme le souligne d'ailleurs la double acception du terme parfumeur du Dictionnaire de Furetière, désignant aussi bien celui «Qui parfume " que celui " qui vend des parfums, des choses parfumées $»^{32}$.

Cependant, outre les parfums qui leur sont appliqués, ces objets ont aussi la particularité de présenter des couleurs variées, d'être brodés d'or ou d'argent, rehaussés de dentelles, bref d'associer dans leur conception plusieurs registres de la technique et de pouvoir être ajustés les uns par rapport aux autres en de véritables systèmes, extensifs ou en abîme, ce qui leur confère à plus d'un titre et de manière performative le rôle de parure du corps, fussent-ils éloignés de lui ${ }^{33}$. Ils participent ainsi d'une conception esthétique englobante, dans l'espace et dans le temps, qui fait entrer en résonance le mobilier, les vêtements et les accessoires, comme le révèlent quelques passages du Mercure Galant et qui, du matin au soir, comme le souligne le diptyque toilettes/déshabillés, contribue à entretenir, à valoriser et à mettre en scène le corps aristocratique dans l'univers domestique. Ces usages témoignent encore de l'existence pérenne d'une culture singulière de la prise en charge du corps dans l'espace curial qui unit plusieurs artisans et/ou officiers, comme le souligne, auprès des nombreux barbiers, la présence de plusieurs tapissiers parmi les valets de chambre de Louis XIV ${ }^{34}$.

31. Pérez, 2007, p. 246.

32. Furetière, 1690, t. 2, p. 261.

33. Lanoë, 2013, p. 25-26.

34. Cavallo, 2007 ; Da Vinha, 2004, p. 53. 


\section{La ganterie, spécialité de la dynastie}

Parmi tous les articles parfumés, les gants occupent une place prédominante dans les trois premiers inventaires de l'Orangerie royale. Parfumé ou non, cet accessoire est, en effet, essentiel à la construction des apparences à la cour depuis le $\mathrm{XVI}^{\mathrm{e}}$ siècle, comme élément indispensable du vêtement, mais aussi par la gestuelle qu'il s'agit de mettre en ouvre pour les enfiler ou les ôter, suggérée par nombre de portraits qui représentent leur sujet avec une main gantée et l'autre à nu. L'importance des gants dans la culture de cour et dans les compétitions économiques de la mode européenne est aussi soulignée par la place qu'ils occupent dans les présents que les souverains s'échangent entre eux au XVII ${ }^{\mathrm{e}}$ siècle $^{35}$. Prisés par douzaines de paires, ils représentent près de $60 \%$ de la valeur du stock de l'Orangerie royale en 1686 et en 1711, pour tomber à $36 \%$ en 1732. Leur valeur marchande et symbolique est soulignée par le classement rigoureux dont ils sont l'objet dans ces inventaires, selon la nature de la matière première (chamois, cabron, mouton, chien et castor), leur qualité, identifiée par des références à des nomenclatures d'origine (gants de Blois, d'Avignon, de Provence), les techniques de leur décoration et de leur apprêt, leur couleur, leur parfum enfin, ce qui, en ces premières années du XVIII ${ }^{\mathrm{e}}$ siècle encore, en fait au moins autant des accessoires d'une construction olfactive que visuelle des apparences.

En effet, les Gallois/Huet ont aussi acquis leur réputation pour la qualité de leurs gants parfumés, au jasmin, et surtout à la fleur d'oranger, dont ils semblent s'être fait une spécialité, fondée sur de véritables savoirs. Précisément, les inventaires de ces gants, dont deux sont établis par l'artisan lui-même, font régulièrement état de l'année de fabrication, précisent lorsqu'ils sont " vieux » ou au contraire « des plus nouveaux », toutes choses qui révèlent une connaissance empirique de la fragilité de ces marchandises, dont la valeur dépend essentiellement de parfums, par définition volatils et éphémères dans le temps. Surtout, certains de ces gants portent une marque, apposée semble-il sur l'article lui-même de la main du fabricant, " $\mathrm{OC}$ » ou « $\mathrm{OB}$ » qui identifient respectivement l'orange commune et l'orange bigarade, employées de manière distincte pour la confection, les fleurs de la seconde permettant d'obtenir un parfum plus intense, des gants plus odorants et donc plus chers (Fig. 4).

35. Rivière, 2019, texte non publié. 


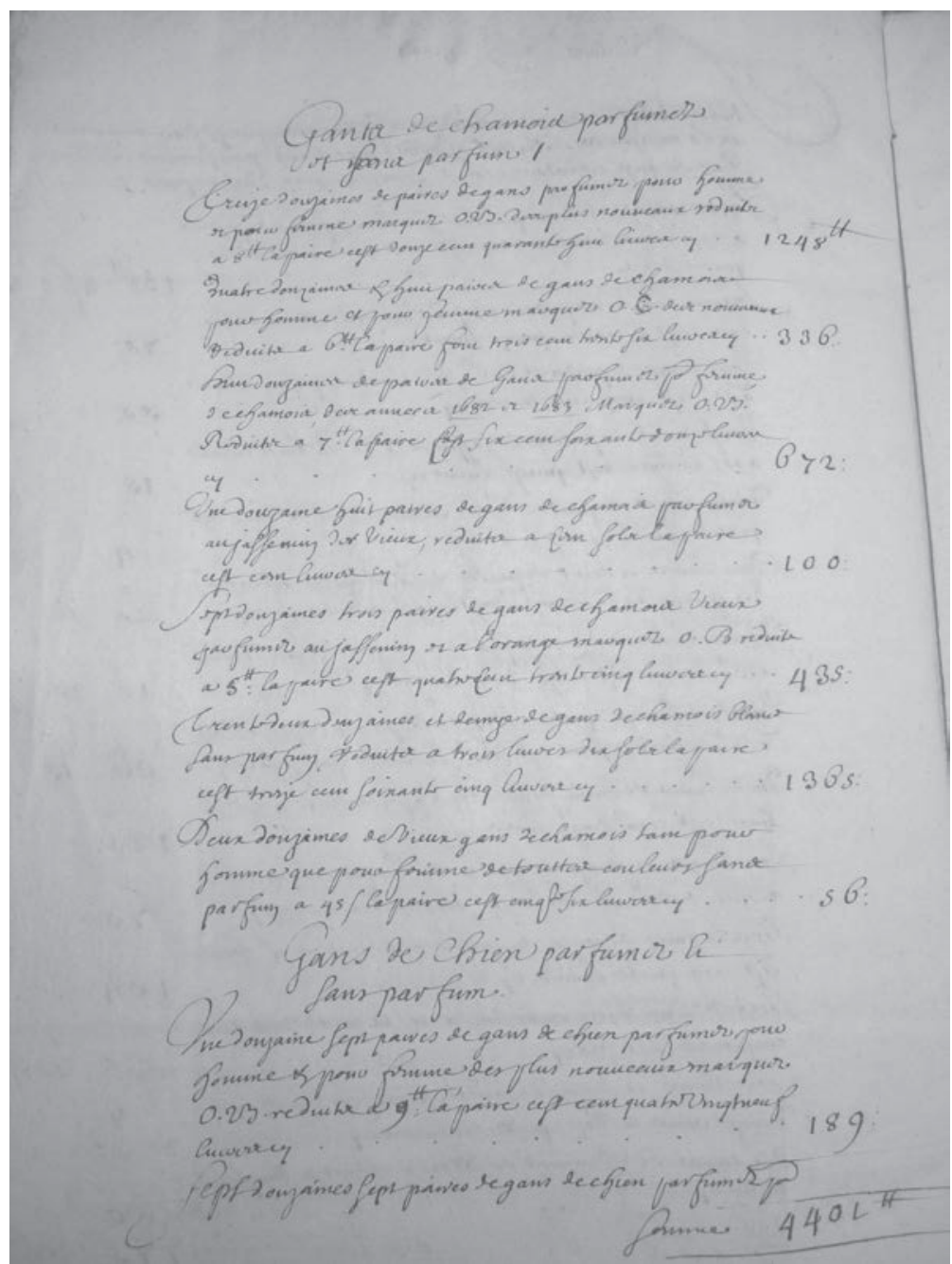

Fig. 4. - Extrait de l'inventaire dressé par Michel Gallois,

\section{2 juillet 1686}

AN, MC XCVII/72, fol. 4.

Archives nationales, photographie Catherine Lanoë

Au cours du XviII ${ }^{\mathrm{e}}$ siècle, cependant, comme l'avaient déjà laissé entrevoir les descriptions des modes du Mercure Galant à la fin du siècle précédent, l'usage des gants parfumés décline, tout comme celui des gants à rubans ou à franges d'ailleurs, au profit de simples gants blancs et surtout de gants blancs glacés. Mieux, en 1732, le Mercure de France annonce la disparition 
prochaine de ces accessoires et, en effet, nombre de gantiers-parfumeurs parisiens de la seconde moitié du siècle n'en proposent plus à la vente ${ }^{36}$. Les patrons de l'Orangerie royale ne sont pas de ceux-là. Au contraire, tout en épousant le déclin relatif de la demande et les nouvelles tendances de la mode, ils restent en mesure de fournir à leur clientèle de tels articles jusqu'à la fin du siècle. De manière signifiante, l'une des clauses additionnelles au contrat de bail de 1751, entre Marie-Louis Quentin de Champlost, valet de chambre du roi, et François Huet stipule que les preneurs s'obligent " fournir audit sieur bailleur pour chacune des neuf années une douzaine et demie de paires de gants blancs d'homme et une douzaine pareille pour femme ", tandis que Marie-Fortunée d'Este, princesse de Conti, fait encore l'acquisition de plusieurs centaines de paires chez Claude-François Prévost durant la seule année 1770, marquée il est vrai par les festivités liées au mariage du Dauphin et de Marie-Antoinette ${ }^{37}$.

À n'en pas douter, le statut de marchand parfumeur du roi et de la cour que détiennent, de longue date, les représentants de la dynastie Gallois/Huet explique que les marchandises de ganterie aient été si pérennes dans leurs stocks, de même qu'ils furent toujours en mesure de proposer à leur clientèle les formes primitives du rouge. Ici encore les actes de la pratique des artisans permettent de nuancer certaines analyses, que biaise parfois l'exploitation exclusive des sources publicitaires et des journaux de mode, toujours tentés de valoriser la nouveauté. Le marché de la cour au XvıII ${ }^{\mathrm{e}}$ siècle, en effet, présente un certain nombre de singularités par rapport à celui de la capitale. Certes, des mouvements de mode, propres à la vie aulique - telle par exemple la vogue des déshabillés (il s'agit ici d'une sorte de robe de chambre) ou celle des gants "à la siamoise ", initiée après la célèbre visite des ambassadeurs en 1686 - ou venus de la ville y sont sensibles, mais la consommation des biens de l'apparence y est aussi marquée par certaines pesanteurs, voire certains archaïsmes, liés à la tradition, au respect de l'Étiquette, au service du roi ${ }^{38}$. Ainsi, par exemple, le " grand habit » féminin et les robes à panier, auxquels sont précisément associés des gants, restent de mise jusque dans les années 1780 , en dépit des récriminations de celles qui

36. Le Mercure de France, février 1732, p. 210 ; Lanoë, 2008, p. 371-373.

37. AN, ET. CVIII/496 ; Chatenet-Calyste, 2013, p. 136.

38. Chrisman-Campbell, 2009, p. 222. 
les portent, tandis qu'au contraire, de l'aveu même de certains marchands, les indiennes de coton ont du mal à s'y imposer ${ }^{39}$.

\section{La révolution des apparences : le visible et l'implicite}

Pour autant que toutes ces marchandises de parfumerie demeurent encore dans le stock de la boutique, les inventaires de 1754 et 1789 révèlent bien des changements, en lien d'une part avec le développement d'une clientèle qui n'appartient plus seulement à l'univers curial et d'autre part avec les inflexions des sensibilités qui, tout à la fois, affectent la construction olfactive des apparences et assurent la promotion d'artifices plus visuels, ainsi que celle de produits d'hygiène du corps.

En 1754, le stock de François Huet fils témoigne de la diversité croissante des marchandises. Parmi les essences et les esprits de parfums dominent les fragrances fraîches du citron, de la bergamote ou de la lavande, privilégiées désormais et qui attestent l'abaissement du seuil de tolérance olfactive identifié par Alain Corbin ${ }^{40}$. À leurs côtés, les cosmétiques pour le visage, tels que les rouges, et pour les cheveux, pommades et poudres, occupent une place de choix. Surtout, l'importance de ces produits dans la fabrique des apparences est accentuée par les nombreux accessoires de leur transport et/ou de leur application - boîtes, étuis, nécessaires, corbeilles, éponges, brosses à rouge, soufflets, masques et houppes à poudre, dont les prix s'inscrivent dans une large gamme. Ainsi, alors que les plus luxueux d'entre eux laissent entrevoir l'aristocratique élégance des nouveaux gestes incorporés de la toilette, des sociabilités mondaines plus mobiles et parfois éloignées de la cour - que l'on peut rapprocher de l'engouement pour la portativité des objets en tous genres d'ailleurs ${ }^{41}$ - d'autres accessoires, plus modestes, soulignent les appropriations populaires des gestes de la beauté, qui ne fonctionnent pas seulement sur le principe de l'imitation des grands. En 1789, la composition du stock de Claude-François Prévost confirme cette tendance. Elle fait aussi la part belle à des articles qui ne sont pas nécessairement nouveaux, mais dont la diversité et le cumul témoignent

39. Hilaire-Pérez et Vaisbrot, 2017. Nous remercions les auteurs qui ont bien voulu nous faire partager cette information.

40. Corbin, 1982.

41. Bernasconi, 2015. 
de la diffusion de nouvelles règles d'hygiène et d'entretien du corps, que certaines institutions médicales entendent d'ailleurs promouvoir à grande échelle. Racines, brosses et éponges pour les dents soulignent les progrès de l'hygiène dentaire que Colin Jones a bien mis en valeur, tandis que la présence renforcée et conjuguée des éponges et des savonnettes révèle combien les pratiques de la toilette, autrefois partiellement sèches, ont réapprivoisé l'usage de l'eau.

Dans cette même temporalité, l'accumulation de ces articles dans le fonds de boutique des deux derniers représentants de la dynastie contribue aussi à flouter l'identité du gantier-parfumeur d'antan au profit de celle du marchand, à dévoiler les ramifications croissantes des structures de la production des articles de beauté, en spécialités diverses, en réseaux d'approvisionnement et de sous-traitance complexes. Joignant à son titre de " parfumeur ordinaire du roi » celui de " marchand mercier », Jean-Daniel Vigier, dont la réussite est éclatante dans les années 1760-1770, illustre à merveille la diffraction qui affecte le secteur, l'inscription du métier dans le marché plus que dans l'atelier, selon les mots de Carolyn Sargentson, mais les Gallois/Huet aussi s'y sont adaptés, comme le révèle la transformation de leur approvisionnement ${ }^{42}$. Ainsi, alors que François Huet père n'avait d'autres fournisseurs que quelques parfumeurs du sud de la France, des gantiers parisiens, de Blois ou de Grenoble auxquels s'ajoutaient un bonnetier, un épicier et un rubanier, les dettes passives de son fils, quarante ans plus tard environ, se répartissent sur des artisans plus nombreux et plus divers : ouvriers en gants ou en mitaines, perruquiers, marchands de pommade, papetiers, faïenciers, mercier, gantier-parfumeurs spécialisés.

\section{Les parfums et les couleurs de la poudre}

Parmi tous les produits qui constituent le stock des patrons successifs de l'Orangerie royale, la poudre pour les cheveux peut être considérée comme emblématique de l'évolution de la fabrique des apparences, des sensibilités qu'elle suppose et qu'elle recompose en même temps, au long d'un siècle qui court du règne de Louis XIV jusqu’à la Révolution. Elle donne lieu au développement d'un vaste marché, à l'émergence d'un débat public et

42. Herda-Mousseaux, 2018, p. 136. 
contribue, elle aussi, à reconfigurer partiellement le visage du métier de gantier-parfumeur.

\section{Les poudres d'odeur}

Les poudres ne sont pas une marchandise nouvelle à la fin $\mathrm{du} \mathrm{XVII}{ }^{\mathrm{e}}$ siècle et, au contraire, elles sont déjà l'objet d'un " grand commerce » dans la capitale $^{43}$. L'ouvrage de Simon Barbe révèle que, comme ingrédient ou comme produit fini, elles permettent la composition de l'univers domestique parfumé décrit plus haut, soit qu'elles servent à imprégner de leur parfum divers objets, soit que, mêlées à des gommes, elles entrent dans la confection de pastilles ou de petits objets à brûler, soit enfin qu'elles soient contenues dans des sachets. Le stock de Michel Gallois en 1686 est précisément constitué de plusieurs produits - on ne saurait dire matières premières tant certaines d'entre elles sont déjà complexes - qui entrent dans la fabrication de ces poudres. Ainsi, la poudre d'iris qu'il conserve se compose à partir du «bâton » de la plante, c'est-à-dire du rhizome qui, une fois écrasé, exhale un parfum naturel de violette, tandis que la poudre de Chypre est un assemblage mixte, non stabilisé, dont le nom n’indique pas une origine géographique : l'ingrédient essentiel est ici la mousse qui croît sur les branches de chênes, séchée, pilée puis diversement parfumée avec des fleurs de jasmin, de rose muscade ou bien de la civette et du musc, mais son appellation pourrait renvoyer au ciperi italien (prononcé Tchi), c'està-dire à l'iris de Florence, élément de sa composition selon d'anciennes recettes ${ }^{44}$.

Pour autant, dès les années 1670-1680, avec le développement du port des longues perruques impulsé par Louis XIV, certaines de ces poudres, classées d'ailleurs en un véritable "Traité » dans l'ouvrage de Simon Barbe, ont aussi pour fonction nouvelle de nettoyer et de parfumer les chevelures, sinon même de les colorer partiellement, qu'elles soient naturelles ou postiches ${ }^{45}$. Évoqués plus haut, Jean et François Quentin fréquentent la capitale et en particulier le quartier Saint-Honoré, au titre de leur charge de barbier et de perruquier de Louis XIV. Chargés de la toilette royale jusqu'en 1690 et précisément de l'achat des poudres et des peignes, ils ne peuvent avoir

43. Blegny, 1692, p. 97.

44. Le Fournier, 1992, p. 36-37.

45. Barbe, 1992 [1699], p. 208-228. 
ignoré l'existence de l'Orangerie royale, tenue par un "parfumeur ordinaire du roi ", et, sans doute, du temps de Michel Gallois et de François Huet père, s'y approvisionnent-ils en différents articles, parmi lesquels figurent justement "poudre d'odeur» et « corps de poudre ${ }^{46}$. Quelque quarante ans plus tard, l'acquisition de la parcelle sur laquelle se trouve la boutique par Jean Quentin, leur descendant, atteste la réputation pérenne de la qualité des marchandises de l'Orangerie royale. Elle promet, sans doute, à son acheteur divers bénéfices matériels, mais elle souligne aussi que l'entretien et la prise en charge du corps du roi structurent fortement les comportements du personnel commensal et forgent une culture qui unit celui-ci aux artisans de l'apparence ${ }^{47}$.

\section{La poudre à poudrer}

Comme le révèlent les stocks des successeurs de Michel Gallois, les usages de la poudre connaissent, en effet, une véritable inflexion dès les premières années du XviII siècle. Dans son Parfumeur royal de 1699, Simon Barbe avait déjà signalé l'existence de " corps de poudre " d'amidon ou bien de " poudres d'amidon » - l'amidon étant confectionné à partir de différentes issues du blé après la mouture. Cette matière première présente selon lui l'avantage de recevoir, mieux que toute autre substance, le parfum de la fleur d'oranger ou celui du jasmin, tout en offrant sans doute la possibilité de confectionner des poudres capables d'éclaircir les chevelures et, en cela, de répondre aux exigences de la mode ${ }^{48}$. Ainsi, de manière presque subreptice, d'artifice olfactif qu'elle était, la poudre pour les cheveux devient aussi un artifice visuel, destiné à blanchir les chevelures et sa consommation s'accroît dans l'univers curial. Dès 1711, le stock de François Huet père compte ainsi près de 2000 livres pesant de poudres diverses, dont près de 900 parfumées à la fleur d'oranger pourraient avoir été confectionnées d'amidon et vouées à éclaircir les chevelures. Vingt ans plus tard, l'état du fonds de boutique que Geneviève de Peyras dresse elle-même pour son fils ne permet plus le doute. La parfumeuse a soin de distinguer « les poudres d'odeur ", parmi lesquelles elle compte près de 900 livres pesant de poudre de fleur d'oranger, de la matière première principale, l'amidon, représen-

46. Da Vinha, 2009, p. 229-230 ; Da Vinha, 2018, p. 191-192.

47. Cavallo, 2007.

48. Barbe, 1992 [1699], p. 210. 
tant à lui seul quelque 2000 livres pesant. Elle fait également figurer un état particulier des houppes de soie et de cygne, de taille et de qualité différentes, dont la seule présence atteste les gestes d'application d'un artifice voué à couvrir l'ensemble des chevelures et donc devenu visuel, tout autant qu'olfactif ${ }^{49}$.

Ainsi que cela a été démontré ailleurs, la consommation de ce que l'on nomme désormais la "poudre à poudrer " s'envole au XvIII ${ }^{\mathrm{e}}$ siècle, quoiqu'elle donne lieu très tôt à de vives critiques de la part du menu peuple - puisque la production de l'artifice contribuait à lui retirer partiellement le pain de la bouche - et aussi à l'éclosion d'un débat d'économie morale ${ }^{50}$. Cette consommation est portée par l'appropriation de ses usages par les catégories intermédiaires, voire populaires, de la capitale, mais encore à partir des années 1770 par la nécessité de faire disparaître les traces de la construction des coiffures des dames, démesurées en hauteur comme en largeur, et dans lesquelles il entrait coussins, postiches de cheveux ou de crin, épingles, pommades... De fait, dans ses Souvenirs, la marquise de la Tour du Pin rapporte qu'une seule livre pesant pouvait être répandue sur une chevelure féminine à l'occasion d'une soirée à la cour, tandis que la consommation mensuelle d'un aristocrate avoisinait les 10 livres ${ }^{51}$.

De cette consommation croissante, les deux derniers inventaires de la dynastie témoignent assurément. Sur les quelque 4700 livres tournois que représentent le stock de marchandises et les ustensiles de fabrication chez François Huet en 1754, 600 livres environ sont constituées par l'amidon, les poudres d'odeur ou à poudrer, les accessoires divers de leur application ou de leur transport, les tonneaux, les tamis et le moulin qui servent à les confectionner et à les conserver. La présence de la poudre est plus accusée encore dans la boutique que Claude-François Prévost dirige en 1789, où ce segment de production, avec ses 1600 livres pesant de poudre et d'amidon, ses 350 livres de poudre passée aux fleurs et 300 livres purgées... représente, en quantité et en valeur, l'essentiel des marchandises et des ustensiles. L'investissement dont il est l'objet se donne aussi à voir dans divers aménagements, identifiables çà et là, et surtout dans la capacité du dirigeant de l'entreprise à adapter ses savoirs techniques, ce qui 
contredit toutes les accusations de routine et de refus de l'innovation portées par l'historiographie traditionnelle de l'artisanat. Ainsi, afin d'écraser et de tamiser au plus fin l'amidon et de produire de la poudre en grande quantité, ce que ne permettaient pas les mortiers et les pilons d'antan, le parfumeur est équipé "d'un moulin » (parfois appelé bluteau), c'est-à-dire d'une machine, contenue en un coffre de bois et actionnée par le mouvement tournant d'une manivelle, qui synthétise les fonctions du moulin qui écrase à celles du bluteau qui affine ${ }^{52}$. Eu égard à l'exiguïté de la boutique et aux nouveaux aménagements dont elle a été l'objet, celui-ci, long de quatre pieds (environ 1,3 mètre), ne peut y être installé sans encombrement ni blanche envolée de fécule dommageable pour le mobilier et la clientèle que l'on reçoit, mais il demeure dans "le magasin au rez-de-chaussée ", c'està-dire dans l'arrière-boutique, prêt à servir. Surtout, le parfumeur dispose de « deux lanternes ", c'est-à-dire de deux exemplaires de la pièce maîtresse de la machine, puisqu'il s'agit de la partie tournante du mécanisme qui contient l'arbre central, sur lequel sont fixées des branches de tôle de fer hérissées qui griffent l'amidon, le tout étant revêtu d'une soie qui le tamise. Lalternance de cette pièce lui permet l'entretien régulier de sa machine 342 - réaffutage de la tôle, changement de la soie - et lui donne la possibilité de garantir à sa clientèle la finesse et la volatilité du produit ${ }^{53}$.

\section{La couleur en héritage}

L'inventaire du stock de la boutique de Claude-François Prévost en 1789 laisse aussi entrevoir une évolution ultime de la poudre, que l'" Art du Parfumeur » de l'Encyclopédie Méthodique ou certains journaux de mode ne manquent pas de souligner, voire d'encourager, et qui attestent les inflexions sensibles et naturalistes des constructions de l'apparence à la fin du XVIII ${ }^{\mathrm{e}}$ siècle. Ainsi, les précisions à propos de la poudre concernent désormais autant la couleur que le parfum, et celle-ci est parfois mise en valeur dans des contenants de verre, telles ces « 77 livres de poudres de différentes couleurs et odeurs dans différentes boîtes et bocaux, prisées 200 livres » chez le dernier patron de l'Orangerie royale. Deux ans auparavant, le Magasin des modes avait ainsi recommandé aux élégantes de renoncer à la poudre blanche qui "durcit et défigure », de privilégier une couleur naturelle, faite 
de subtils mélanges de teintes brunes, grises, blondes, roses ou rousses, ce que, sans doute, la clientèle de Claude-François Prévost pouvait effectuer en fréquentant sa boutique ${ }^{54}$.

La maîtrise des savoirs de la couleur par les gantiers-parfumeurs que révèle, dès la fin du grand siècle, la confection des poudres - Michel Gallois ne dispose-t-il pas déjà de poudre de polleville que Simon Barbe déclare de " couleur un peu rougeâtre " ? - ne peut donner lieu à beaucoup de surprise, surtout lorsqu'il s'agit des Gallois/Huet ${ }^{55}$. En effet, beaucoup plus que d'autres de leurs confrères, ces derniers ont précocement développé une sensibilité aux couleurs, du fait de l'approvisionnement curial qu'ils ont assuré et la connaissance des goûts et des attentes de leur clientèle aristocratique. En témoignent en particulier les deux inventaires de marchandises dressés par Michel Gallois en 1686 et par Geneviève de Peyras en 1732, dans lesquels abondent les précisions chromatiques, aux côtés des mentions olfactives, contrastant avec les descriptions notariales plus neutres sur ce point. Il en va ainsi, chez le premier, de l'expression « couleur de cerise " qui caractérise certaines toilettes et déshabillés, bien distinguée de "l'incarnat ", et si Geneviève de Peyras liste parmi ses marchandises des peaux et des gants parfumés " de toutes couleurs ", blancs ou jaunes, elle se fait plus précise lorsqu'il s'agit de ces mêmes articles, évoquant encore la "couleur de cerise ", mais aussi la " couleur de rose " (Fig. 3), une expression que La Fontaine avait utilisée en 1669 pour décrire le plumage des cormorans de la ménagerie de Versailles, mais qui fait flores sous la plume des libertins dans les années $1730^{56}$. Naturellement, une telle sensibilité est aussi un héritage de la tradition de la ganterie, avec les opérations de " mise en couleur " des peaux et des gants, que les Gallois/Huet ont longtemps préservé. En effet, si les matières premières nécessaires à la fabrication des teintures peuvent être communes à la préparation d'autres types de marchandises, ainsi le talc, l'amidon, la cochenille... utilisés pour la confection de la poudre ou de fards, les patrons de l'Orangerie royale disposent jusqu'en 1754, au moins, de plusieurs marbres et molettes qui leur permettent d'écraser les différents pigments pour confectionner ces

54. Lanoë, 2008, p. 271.

55. Barbe, 1992 [1699], p. 225.

56. La Fontaine, 1990, p. 44-45 (1669). Nous remercions Aurélia Gaillard, professeur de littérature française du XVIII ${ }^{\mathrm{e}}$ siècle à l'Université Bordeaux Montaigne, qui nous a signalé ces références. On verra son article "Le rose des Lumières ", dans Lumières n 36, à paraître en 2020. 
couleurs. La ganterie fait d'eux des pratiquants de la couleur, autant peutêtre que des parfums, tandis que la volonté de satisfaire les consommateurs exigeants et connaisseurs de la cour et de la ville stimule de l'un à l'autre de ces arts, d'une génération à une autre, et dans de multiples sens, les transpositions techniques et les adaptations terminologiques constitutives de leurs savoirs.

$\mathrm{Au}$ terme de cette réflexion, menée à hauteur d'homme, il apparaît une fois encore combien les actes notariés et les inventaires après décès en particulier sont des sources essentielles, encore trop peu exploitées, tant pour l'histoire sociale et celle des sensibilités que pour l'histoire des techniques et celle des savoirs. Les actes de la pratique de la dynastie Gallois/Huet permettent, en effet, d'identifier un modèle spécifique d'exercice de la ganterie-parfumerie, inscrit, tout au long de son siècle d'existence, dans les logiques particulières de l'approvisionnement de la cour que les dirigeants de l'entreprise parviennent à associer aux caractéristiques nouvelles du marché de l'apparence, marqué par le renouvellement rapide des articles, leur démocratisation relative et bientôt la promotion de gestes d'hygiène censés embrasser et policer l'ensemble de ces corps. Le profil de l'artisan commensal, comme le modèle de cour et sa culture des apparences d'ailleurs, sont ainsi ébranlés et adaptés, mais ils résistent et ces négociations, entre l'ancien et le nouveau, s'avèrent pleinement représentatives de la civilisation des Lumières. À une autre échelle, l'analyse de ce corpus donne aussi la possibilité d'entrevoir comment s'articulent culture de l'olfactivité et culture de la couleur dans la France de l'Ancien Régime et de quelles façons les gantiers-parfumeurs de ce temps sont passés maîtres de savoirs qui construisent l'une et l'autre.

\section{Sources}

\section{Sources manuscrites}

Archives nationales, Minutier central.

ET. XCVII/72 ; ET. XLIX/456 ; ET XLIX/547 ; ET. XC/382 ; ET. XLIII/556 ; ET. LIX/128; ET. CVIII/496; ET. LVI/241-242 ; ET. CXV/256 ; ET. XX/556; ET. XLVI/419 ; ET. LXIV/301 ; ET. XLIX/598 ; ET. XC/435 ; ET. LVI/213. Archives nationales, série Z1J. Greffiers des bâtiments. Z1J/623.

Archives départementales de Haute-Saône. Amance, (1737-1747), EC_12EDEPOT5, fol. 61. 


\section{Sources imprimées}

"Art du parfumeur ", Encyclopédie Méthodique, Arts et métiers mécaniques, t. vI, Paris, Panckoucke, Liège, Plomteux, 1789, p. 1-60.

Barbe Simon, Le parfumeur royal [1699], Klincksieck, Paris, 1992.

Blegny Nicolas (de), Le livre commode contenant les Adresses de la ville de Paris..., chez la veuve de Denis Nion, Paris, 1692.

Essai sur l'almanach général d'indication d'adresse personnelle et domicile fixe des six corps..., chez la veuve Duchesne, Paris, 1769.

Furetière Antoine, Dictionnaire universel..., 1690, [édition Gallica], t. 2, "Parfumeur ".

La Fontaine Jean, Psyché, GF-Flammarion, Paris, 1990 [Paris, Claude Barbin, 1669].

Le Fournier André, La décoration d'humaine nature et ornement des dames [1541], Klincksieck, Paris, 1992.

L'Esprit des modes au Grand siècle, Édition de Corinne Thépaut-Cabasset, Éditions du CTHS, Paris, 2010.

\section{Bibliographie}

Arizzoli-Clémentel Pierre, Gorguet Ballesteros Pascale (dir.), Fastes de cour et cérémonies royales. Le costume de cour en Europe, 1650-1800, RMN, ParisVersailles, 2009.

BÉguin Katia, Lyon-Caen Nicolas, " "Dans la chaleur des enchères". Adjudications et prix des immeubles à Paris aux XVII ${ }^{\mathrm{e}}$ et XVIII ${ }^{\mathrm{e}}$ siècles ", Revue d'histoire moderne et contemporaine, 2018, n 65, p. 144-166.

Belfanti Carlo Marco, Histoire culturelle de la mode, Institut Français de la mode/Regard, Paris, 2014.

Bernasconi Gianenrico, Objets portatifs au siècle des Lumières, Éditions du CTHS, Paris, 2015.

Braudel Fernand, Civilisation matérielle, Économie et Capitalisme, XV'-XVIII siècle, t. 1, Les structures du quotidien, A. Colin, Paris, 1979.

Briot Eugénie, "Normes et pratiques du corps parfumé à la cour de France ", dans Da Vinha Mathieu, Lanoë Catherine, Laurioux Bruno (dir.), Cultures de cour, Cultures du corps. XVI'-XVIII siècles, Presses de la Sorbonne, Paris, 2011, p. 257-272.

Briot Eugénie, "Jean-Louis Fargeon, fournisseur de la cour de France, arts et techniques d'un parfumeur du XVIII ${ }^{\mathrm{e}}$ siècle, dans Lanö̈ Catherine, MoulinIER Laurence (dir.), Corps parés, Corps parfumés, Artefact. Techniques, histoire et sciences humaines, $\mathrm{n}^{\circ} 1,2013$, p. 167-177. 
Briot Eugénie, La Fabrique des parfums, Naissance d'une industrie de luxe, Vendémiaire, Paris, 2015.

Bruna Denis, Demey Chloé (dir.), Histoire des modes et du vêtement. Du Moyen Âge au XXI siècle, Textuel, Paris, 2018.

Carbonnier Youri, Maisons parisiennes des Lumières, PUPS, Paris, 2006.

Castres Astrid, Brodeurs et chasubliers à Paris au XVI siècle, thèse de l'École Pratique des Hautes Études, Paris, 2016, 2 vols.

Cavallo Sandra, Artisans of the body in early Modern Italy, Manchester University Press, Manchester, 2007.

Chatenet-Calyste Aurélie, « La parure de Marie-Fortunée d'Este, princesse de Conti (1731-1803). Une médicalisation du paraître? ", dans Lanoë Catherine, Moulinier Laurence (dir.), Corps parés, Corps parfumés, Artefact. Techniques, histoire et sciences humaines, $\mathrm{n}^{\circ} 1,2013$, p. 133-152.

Coquery Natacha, L'Hôtel aristocratique. Le marché du luxe à Paris au XVIII siècle, Publications de la Sorbonne, Paris, 1998.

Coquery Natacha (dir.), La Boutique et la ville. Commerces, commerçants, espaces et clientèles, $X V T^{e}-X X^{e}$ siècle, Publications de l'Université François Rabelais, Tours, 2000.

Coquery Natacha, Tenir boutique à Paris au XVIII siècle, Éditions du CTHS, Paris, 2011.

Corbin Alain, Le Miasme et la jonquille, Aubier Montaigne, Paris, 1982.

Chrisman-Campbell Kimberly, "Le grand habit et la mode en France au Xviir ${ }^{\mathrm{e}}$ siècle ", dans Arizzoli-Clémentel Pierre et Gorguet Ballesteros Pascale (dir.), Fastes de cour et cérémonies royales. Le costume de cour en Europe, 1650-1800, RMN, Paris-Versailles ; 2009, p. 222-225.

Da Vinha Mathieu, Les Valets de chambre de Louis XIV, Perrin, Paris, 2009, [1 $1^{\text {re }}$ éd. Perrin, 2004].

Da Vinha Mathieu, Lanoë Catherine, Laurioux Bruno (dir.), Cultures de cour, Cultures du corps. XVI-XVIII siècles, Presses de la Sorbonne, Paris, 2011.

Da Vinha Mathieu, Masson Raphaël (dir.), Versailles. Histoire, Dictionnaire, Anthologie, Robert Laffont, Paris, 2015.

Da Vinha Mathieu, Au service du roi. Les métiers à la cour de Versailles, Texto,

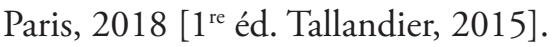

Gaillard Aurélia et Lanö̈ Catherine (dir.), La Couleur des Lumières, Dixhuitième Siècle, n' 51, 2019.

Gayne Mary K., The Wigmakers, The Public and The State: Cultural and Material Production of Eighteenth-Century Hairstyles, thèse de doctorat, Cornell University, 2007.

Gorguet Ballesteros Pascale, "Caractériser le costume de cour : propositions ", dans Arizzoli-Clémentel Pierre et Gorguet Ballesteros Pascale (dir.), Fastes de cour et cérémonies royales. Le costume de cour en Europe, 1650 1800, RMN, Paris-Versailles, 2009, p. 54-69. 
Herda-Mousseaux Rose-Marie (dir.), La Fabrique du luxe. Les marchands merciers parisiens au XVIII' siècle, Catalogue d'exposition, Musée Cognacq Jay, Paris, 2018. Hilaire-Pérez Liliane, Lanoë Catherine, "Pour une relecture de l'histoire des métiers : les savoirs des artisans en France au XviII ${ }^{\mathrm{e}}$ siècle ", in Milliot Vincent, Minard Philippe, Porret Michel (dir.), La grande chevauchée. Faire de l'histoire avec Daniel Roche, Droz, Genève, 2011, p. 357-370.

Hilaire-Pérez Liliane, La Pièce et le geste. Artisans, marchands et savoir technique à Londres au XVIII' siècle, Albin Michel, Paris, 2013.

Hilaire-Pérez Liliane, VAisBrot Bernard, "The Yiddish correspondence of Louis de Berlin, 1775-1776 : presentation of the corpus", École d'été "Revisiting the Early Modern Economic History of the Jews : Sources, Historiography, Questions" (Evelyne Oliel-Grausz dir.), Centre de Recherche français à Jérusalem (CRF), 13 juillet 2017, texte non publié.

Jones Colin, The Smile Revolution in Eigthteenth Century Paris, Oxford University Press, Oxford, 2014.

Jones Jennifer, Sexing La Mode: Gender, Fashion and Commercial culture in Old Regime France, Berg Publishers, Oxford, 2004.

Labreuche Pascal, Paris, capitale de la toile à peindre, XVIII -XIXe siècle, Éditions du CTHS, INHA, Paris, 2011.

LANOË Catherine, La Poudre et le fard. Une histoire des cosmétiques de la Renaissance aux Lumières, Champ Vallon, Seyssel, 2008.

LANOË Catherine, "Les systèmes de parure comme langage technique ", dans Lanö̈ Catherine, Moulinier Laurence (dir.), Corps parés, Corps parfumés, Artefact. Techniques, histoire et sciences humaines, $\mathrm{n}^{\circ}$ 1, 2013, p. 13-31.

LAVERNY Sophie (de), "Le petit commerce commensal face aux corporations des villes jurées au XVII e siècle ", dans Coquery Natacha (dir.), La Boutique et la ville. $X V I^{e}-X X^{e}$ siècle, Publications de l'Université François Rabelais, Tours, 2000, p. 17-30.

LaVerny Sophie (de), Les Domestiques commensaux du roi de France au XVIT siècle, PUPS, Paris, 2002.

Leferme Falguières Frédérique, Les Courtisans : une société de spectacle sous l'Ancien Régime, PUF, Paris, 2007.

Letourmy-Bordier Georgina, Los Llanos José-Louis (de), Le Siècle d'or de l'éventail : du Roi-Soleil à Marie-Antoinette, catalogue d'exposition, Musée Cognac-Jay, 14 novembre 2013- 2 mars 2014, Éditions Faton, Dijon, 2013.

Lheureux Rosine, Une histoire des parfumeurs. France 1850-1910, Champ Vallon, Ceyzérieu, 2016.

LYON-CAEN Nicolas, "L'immobilier parisien au XVIII e siècle. Un marché locatif ", Société française d'histoire urbaine, 2015/2 nº 43, p. 55-70.

Mauss Marcel, "Les techniques du corps ", dans Mauss Marcel, Sociologie et anthropologie, PUF, Paris, 1960 [1 ${ }^{\text {re éd. 1936]. }}$ 
McNeil Peter (dir.), A Cultural History of Dress and Fashion in the Age of Enlightenment (1650-1800), Bloomsbury, Londres, 2017.

Paresys Isabelle, Coquery Natacha (dir.), Se vêtir à la cour en Europe, 14001815, Irhis-Ceges-Centre de recherche du château de Versailles, Villeneuve d'Asq, 2011.

PÉrez Stanis, La Santé de Louis XIV. Une biohistoire du roi-soleil, Champ Vallon, Seyssel, 2007.

Pérez Stanis, "L'eau de fleur d'oranger à la cour de Louis XIV ", dans Lanö̈ Catherine, Moulinier Laurence (dir.), Corps parés, Corps parfumés, Artefact. Techniques, histoire et sciences humaines, $\mathrm{n}^{\circ} 1,2013$, p. 107-115.

Perrot Philippe, Le Corps féminin. Le travail des apparences, XVIII-XIXe siècle, Seuil, Paris, 1984.

RIVIÈre Chloé, "Dire l'identité du prince : parures et joyaux dans les présents diplomatiques au $\mathrm{XVII}^{\mathrm{e}}$ siècle ", communication prononcée au colloque The Gestures of Diplomacy, organisé par Roberta Anderson, Nathalie Rivère de Carles, Toulouse, 30-31 mai 2019.

Roche Daniel, La Culture des apparences. Une histoire du vêtement, XVII -XVIII siècle, Fayard, Paris, 1989.

Sargentson Carolyn, Merchants and Luxury Markets. The Marchands Merciers of Eighteenth-century Paris, Victoria and Albert Museum Editions, Londres, 1996.

Velut Christine, "Le monde intérieur de la boutique : les boutiques de papiers peints à Paris, 1750-1820 ", dans Coquery Natacha (dir.), La Boutique et la ville. Commerces, commerçants, espaces et clientèles, $X V t^{e}-X X^{e}$ siècle, Publications de l'Université François Rabelais, Tours, 2000, p. 277-291.

Vigarello Georges, Le Propre et le sale, Seuil, Paris, 1985.

Walsh Claire, "Shopping et tourisme : l'attrait des boutiques parisiennes au XVIII ${ }^{\mathrm{e}}$ siècle ", dans Coquery Natacha (dir.), La Boutique et la ville. Commerces, commerçants, espaces et clientèles, $X V T^{e}-X X^{e}$ siècle, Publications de l'Université François Rabelais, Tours, 2000, p. 223-237.

\section{L'autrice}

Catherine Lanoë est maîtresse de conférences en histoire moderne à l'université d'Orléans. Sa thèse a été publiée en 2008 sous le titre La Poudre et le fard. Une histoire des cosmétiques de la Renaissance aux Lumières (Champ Vallon). Spécialiste de l'histoire des cosmétiques et de la parfumerie, elle poursuit ses travaux dans le domaine de l'histoire du corps et de la beauté, de sa culture matérielle, de ses représentations et de ses savoirs.Contact : catherine_lanoe@hotmail.com 